Revista Latinoamericana de la Papa 22 (1): 49 - 57

http://www.papaslatinas.org/revista.html

ISSN: $1853-4961$

\title{
Comportamiento de dos variedades de papa bajo estrés hídrico
}

\section{M.C. Bedogni ${ }^{1,2}$, S.B.Capezio ${ }^{2 / *}$ (ex aequo), G. Echaide ${ }^{2}$}

Recibido: $22 / 05 / 2018$

Aceptado: 06/106/2018

Accesible en línea: Junio de 2018

\section{Resumen}

El estrés por sequía es uno de los principales factores limitantes del rendimiento y la calidad de tubérculos en el cultivo de papa. El objetivo del trabajo fue evaluar el comportamiento de dos variedades de papa, Spunta y Newen INTA frente a sequía en condiciones de campo. Se realizaron dos experimentos, uno bajo riego y otro en secano. En cada uno se siguió un diseño experimental de bloques completos aleatorizados con cuatro repeticiones. A partir de los 45 días después de plantación y cada 15 días se midió conductancia estomática en el folíolo terminal, número de tubérculos, peso fresco y seco de follaje y tubérculos. A la cosecha se clasificaron los tubérculos por tamaño, y se calculó el rendimiento total y comercial, el porcentaje de materia seca y se evaluó la aptitud para freír de los mismos. La variedad Newen INTA fue la que mantuvo mayor conductancia estomática frente a sequía. Ambas variedades redujeron el rendimiento total y el rendimiento comercial bajo condiciones de secano. Newen INTA fue la que obtuvo los mayores rendimientos en condiciones de sequía sin diferir estadísticamente de Spunta.

Palabras claves adicionales: Solanum tuberosum, sequía, conductancia estomática, rendimiento.

\footnotetext{
* Autor para correspondencia. Correspondencia electrónica: scapezio@ mdp.edu.ar

${ }^{1}$ EEA INTA Balcarce, Argentina. E-mail: bedogni.maria@inta.gob.ar

${ }^{2}$ Facultad Ciencias Agrarias, UNMdP, Balcarce. Argentina
} 


\section{Performance of two potato varieties under water stress}

\section{Summary}

It is a relevant task for current agricultural research to predict and interpret the influence of climate change on the harmful organisms of crops and to adapt the phytosanitary management. Interpreting the bioclimatic scenarios of Phytophthora infestans, which is the causal agent of late blight of potato in the crops areas in Ciego de Ávila during the period November - April for the years 2025 and 2030 under scenarios (SRES) A2 and B2 was the aim of this work. In the making of the bioclimatic scenarios, were used daily data of accumulated precipitation and the average temperature from the Regional Model PRECISCARIBE. In addition, the biological indexes required by the phytopathogen to develop epiphytics which are considered in the method of "threshold of rains" recommended by the technical instruction of the crop in Cuba were taken into account. The results agree on the existence of not favorable meteorological conditions for the development and evolution of late blight in both 2025 and 2030. Specifically, the scarce occurrence and the low cumulative of precipitations that do not exceed the levels indicated by the threshold line, as well as the incidence of average temperatures above $24{ }^{\circ} \mathrm{C}$. The bioclimatic scenarios obtained for this Oomycetes provide information for future planning and phytosanitary management of the crop as well as for the selection of cultural inputs and services that favor the application of climate-smart agriculture that contributes to the sustainable production of the tuber.

Additional keywords: Solanum tuberosum, drought, stomatal conductance, yield.

\section{Introducción}

La papa (Solanum tuberosum L.) pertenece a la familia de las Solanáceas y su origen se encuentra en América del Sur (Ochoa 1999). Ocupa el cuarto lugar a nivel mundial como cultivo más importante en cantidad producida superado sólo por el maíz, el trigo y el arroz y el sexto lugar como alimento (FAOSTAT 2016). En Argentina, la mayor superficie cultivada de papa y los mayores rendimientos se logran en el Sudeste bonaerense, convirtiendo a esta zona en la principal región productora del país (Argenpapa 2016).

La variedad más difundida en Argentina es Spunta, de origen holandés, que produce altos rendimientos. En el programa de mejoramiento de papa de la EEA Balcarce se han obtenido variedades como Frital INTA, Newen INTA, Calén INTA, Pampeana INTA, entre otras, que si bien no están muy difundidas, algunas presentan un mejor comportamiento que Spunta frente a condiciones adversas (Huarte y Capezio 2013).

El cultivo de papa requiere una temperatura de $20^{\circ} \mathrm{C}$ para crecer vegetativamente, mientras que la temperatura óptima para la tuberización está entre los 14 y $22^{\circ} \mathrm{C}$. La papa requiere entre 400 y $800 \mathrm{~mm}$ de agua, dependiendo de las condiciones agroclimáticas y del manejo del cultivo. Un déficit hídrico durante el ciclo de crecimiento del cultivo de papa trae como consecuencia bajos rendimientos y mala calidad de tubérculos. Cuando se produce un estrés hídrico principalmente durante el período crítico (inicio de la tuberización) es necesario incrementar la eficiencia con la que la papa emplea el agua teniendo en cuenta que la disponibilidad de la humedad en el suelo no descienda del $50 \%$ de la capacidad de 
campo (Ekanayake 1994, Carrillo et al. 2003).

El comportamiento de las distintas variedades de papa ante periodos de estrés hídrico está influenciado por diversos factores morfofisiológicos que le permiten aumentar su tolerancia. Estos factores pueden ser cambios morfológicos en las hojas, mecanismos de osmoregulación, alteraciones en la relación raíz/parte aérea, comportamiento estomático, etc. (Olalla Mañas et al. 2005). Por lo que la tolerancia a sequía es un carácter complejo que requiere de validaciones en diversas condiciones de cultivo y está controlada por varios genes de efectos menores o QTLs muy influenciados por el ambiente (Coleman 2008).

Ante la creciente influencia del cambio climático y debido a que la actual base genética de la papa presenta escasa variabilidad, se hace necesario identificar materiales genéticos con resistencia o tolerancia a sequía. Magaña (2013) caracterizó el comportamiento de varios genotipos de papa frente a sequía en invernáculo, dos de los cuales, Newen INTA y Spunta tuvieron un buen comportamiento en cuanto al vigor, tasa de alargamiento foliar, altura de la planta y contenido relativo de agua. Debido a este resultado preliminar el objetivo de este trabajo fue caracterizar el comportamiento de estas dos variedades frente a déficit hídrico en condiciones de campo.

\section{Materiales y Métodos}

Se realizaron dos ensayos en un lote de la EEA INTA Balcarce ( $37^{\circ} 45^{\prime} 32^{\prime \prime}$ S; $58^{\circ} 0,17^{\prime}$, O) durante la campaña 2014/15.Se utilizaron dos variedades de papa Solanum tuberosum subsp. tuberosum (Spunta y Newen INTA) empleando papa semilla categoría prefundación. Se siguió un diseño en bloques completos aleatorizados con cuatro repeticiones. $\mathrm{La}$ unidad experimental consistió de cuatro surcos de $5 \mathrm{~m}$ distanciados $85 \mathrm{~cm}$ entre sí. La fecha de plantación fue el 15 de noviembre de 2014. Uno de los ensayos se condujo sin aplicación de riego y en el otro se aplicó riego por aspersión durante el desarrollo del cultivo. Se suministraron catorce riegos de $30 \mathrm{~mm}$ cada uno mediante el sistema de rollo con ala regadora. A partir de los 45 días después de plantación (ddp) se midió la conductancia estomática $\left(\mathrm{mmol} / \mathrm{m}^{2} . \mathrm{s}\right)$ (entre las 12:30 - $13 \mathrm{hs}$ ) en el folíolo terminal de la cuarta hoja de una planta elegida al azar de los dos surcos centrales de cada repetición, mediante la utilización de un porómetro marca Decagon. Se hicieron cuatro mediciones a lo largo del ciclo del cultivo (45, 60, 75 y $90 \mathrm{ddp})$. Al mismo tiempo se cosecharon 4 plantas de los surcos 1 y 4 y se clasificaron en raíz, parte aérea y tubérculo; se registró el peso fresco y seco de cada muestra. Al finalizar el ciclo del cultivo se cosecharon los dos surcos centrales con una arrancadora de discos el 27 de marzo de 2015. Se determinó el rendimiento total y el número y peso de los tubérculos, los cuales fueron clasificados por tamaño: menores de $50 \mathrm{~mm}$, entre 50 y $75 \mathrm{~mm}$, entre 75 y $90 \mathrm{~mm}$ y mayores de $90 \mathrm{~mm}$ de longitud mediante la utilización de zarandas. Además se registró el peso de los tubérculos que presentaban pudriciones y defectos externos $\mathrm{Se}$ determinó el rendimiento comercial restándole al rendimiento total el peso de tubérculos menores a $50 \mathrm{~mm}$, el peso de tubérculos con pudriciones y el peso de defectos externos. Se calculó el porcentaje de materia seca a través del método gravimétrico. Se determinó la calidad de las papas fritas en rodajas delgadas asignándole un puntaje en base 
al color, comparándolo con una escala de colores de 1 a 9, siendo de 1-4 (no aceptable, color muy oscuro), de 5-6 (aceptable, color dorado) y de 7-9 (aceptable, color muy pálido) (Colour cards for quality evaluation of potato chips, Wageningen, The Netherlands).

Se calculó el agua disponible en el suelo bajo condiciones de sequía y bajo riego. $\mathrm{Se}$ registraron diariamente las temperaturas, lluvias y evapotranspiración y se realizaron los balances de agua bajo condiciones de secano y riego de acuerdo a Della Maggiora (2011).
El análisis de los datos se realizó mediante análisis de varianza y de medias con el programa R (2013).

\section{Resultados y discusión}

De acuerdo a los balances de agua se evidenció que hubo un déficit hídrico durante el crecimiento del cultivo en condiciones de secano. A partir de los 28 ddp se registró un déficit hídrico que duró hasta el final del ciclo del cultivo. Se registró una precipitación importante a los 82 ddp, que no fue significativa debido al momento del ciclo del cultivo y al déficit hídrico existente (Figura 1).

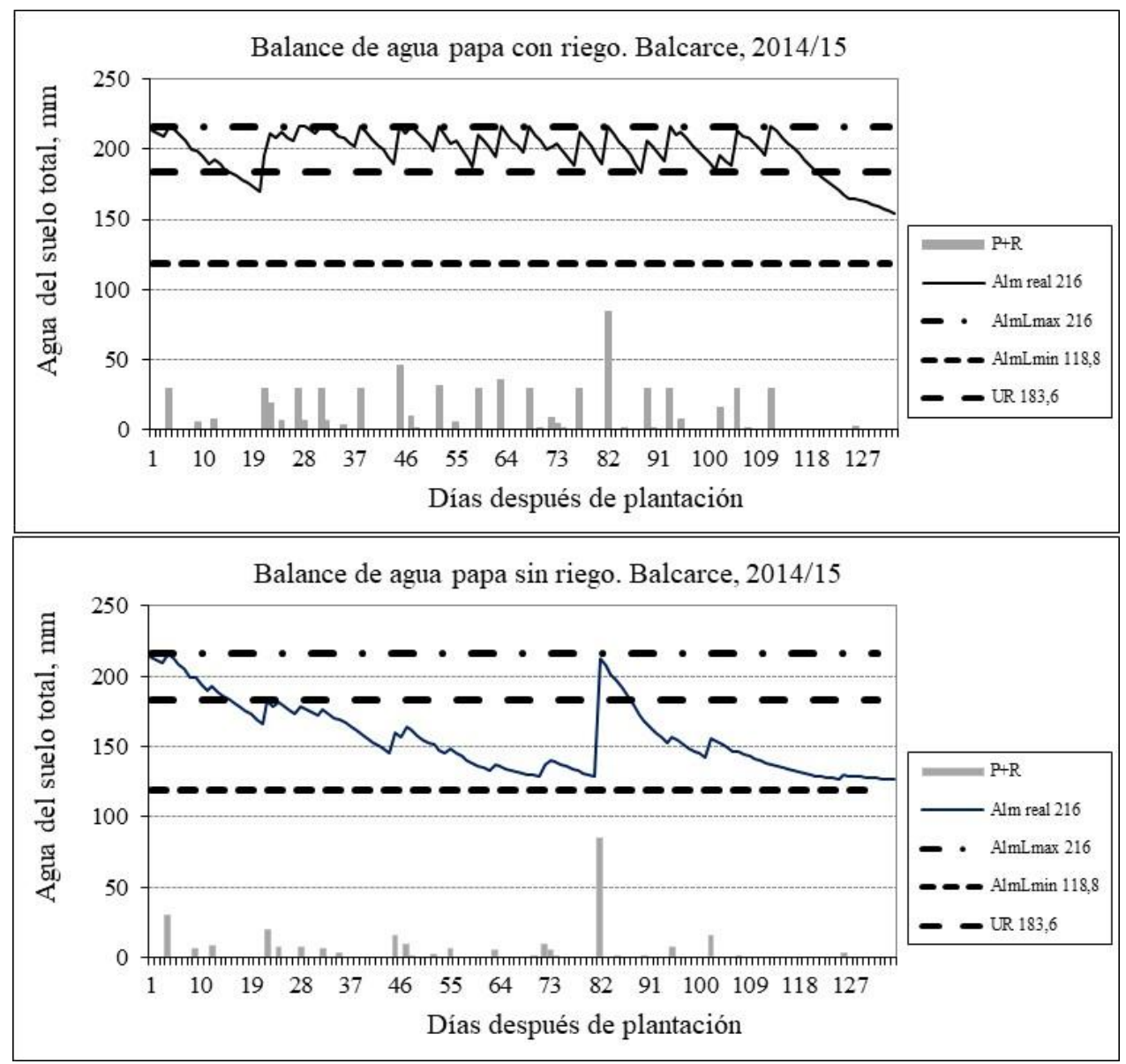

Figura 1. Balance de agua bajo condiciones de secano y bajo riego. Balcarce 2014/15

La variedad Newen INTA presentó mayor conductancia estomática que Spunta en condiciones de estrés y bajo riego durante el ciclo del cultivo. A los 45 y a los 90 
ddp Newen presentó una conductancia significativamente mayor que Spunta. Bajo condiciones de estrés, a los 45 ddp, Newen disminuyó la conductancia un $28 \%$ y Spunta un $65 \%$ mientras que a los 90 ddp la disminución fue de $34 \%$ y $28 \%$ respectivamente (Tabla 1). Esto indicaría diferentes eficiencias en la capacidad para mantener la tasa de intercambio gaseoso bajo condiciones de estrés. Estos resultados concuerdan con Nuñez Barrios et. al. (1989), Pérez et. al. (2008) y Ortega Ortiz (2006) quienes observaron en varias especies vegetales, bajo condiciones de estrés hídrico, una reducción de la conductancia estomática de las hojas. Esta disminución provocaría una reducción en la fotosíntesis, pudiendo producir una pérdida de rendimiento en caso de persistir el estrés a lo largo del ciclo del cultivo.

Tabla 1. Conductancia estomática $\left(\mathrm{mmol} \mathrm{m} \mathrm{m}^{-2} \mathrm{~s}^{-1}\right)$ a $\operatorname{los} 45,60,75$ y 90 ddp bajo condiciones de secano y riego en las variedades Spunta y Newen INTA. Balcarce, 2014/15.

\begin{tabular}{ccccc}
\hline \multirow{2}{*}{ Variedad } & \multicolumn{4}{c}{ Días después de plantación } \\
& 45 & 60 & 75 & 90 \\
\hline Secano & & & & \\
Newen & $934 \mathrm{a}$ & $787 \mathrm{a}$ & $576 \mathrm{a}$ & $717 \mathrm{a}$ \\
Spunta & $488 \mathrm{~b}$ & $747 \mathrm{a}$ & $493 \mathrm{a}$ & $486 \mathrm{~b}$ \\
Riego & & & & \\
Newen & $1299 \mathrm{a}$ & $1542 \mathrm{a}$ & $939 \mathrm{a}$ & $1089 \mathrm{a}$ \\
Spunta & $1428 \mathrm{a}$ & $1434 \mathrm{a}$ & $1028 \mathrm{a}$ & $677 \mathrm{~b}$ \\
\hline
\end{tabular}

Spunta presentó valores más altos de peso fresco de la parte aérea que Newen INTA en ambos ensayos durante todo el ciclo de crecimiento. A su vez los 90 ddp Spunta obtuvo mayor peso fresco de la parte aérea y menor conductancia que Newen INTA en ambos ensayos. Estos resultados coinciden con lo planteado por Martínez y Moreno (1992) quienes concluyeron que hay una estrecha relación entre la conductancia foliar y la fotosíntesis durante un período de estrés hídrico.
Algunas variedades tienen mayor ahorro de agua debido a que poseen una mayor sensibilidad estomática a expensas de la asimilación de carbono y otras variedades mantienen un nivel de apertura estomática para el intercambio gaseoso en los mismos niveles de déficit hídrico. El peso fresco de los tubérculos en condiciones de secano fue mayor en Spunta que en Newen hasta los 90 ddp, siendo solo significativamente mayor a los $60 \mathrm{ddp}$ (Tabla 2). 
Tabla 2. Peso fresco y seco de follaje, peso fresco de tubérculos a los 45, 60, 75 y 90 días después de plantación en dos variedades de papa en condiciones de secano y bajo riego, (muestra de 4 plantas). Balcarce 2014/15

\begin{tabular}{|c|c|c|c|c|c|c|c|c|}
\hline & \multicolumn{8}{|c|}{ Días después de plantación } \\
\hline & \multicolumn{2}{|l|}{45} & \multicolumn{2}{|l|}{60} & \multicolumn{2}{|l|}{75} & \multicolumn{2}{|l|}{90} \\
\hline & Secano & Riego & Secano & Riego & Secano & Riego & Secano & Riego \\
\hline \multicolumn{9}{|c|}{ Peso fresco follaje (gr) } \\
\hline Newen & 1659 a & 1936 a & 1859 a & $2881 \mathrm{a}$ & $1635 \mathrm{a}$ & $4005 \mathrm{a}$ & $1116 \mathrm{a}$ & $2177 \mathrm{a}$ \\
\hline Spunta & 2016 a & 2223 a & $2450 \mathrm{a}$ & $3090 \mathrm{a}$ & 2045 a & $4762 \mathrm{a}$ & $1294 \mathrm{a}$ & $2450 \mathrm{a}$ \\
\hline \multicolumn{9}{|c|}{ Peso seco follaje (gr) } \\
\hline Newen & $175 \mathrm{a}$ & $198 \mathrm{a}$ & $228 \mathrm{a}$ & $326 \mathrm{a}$ & $252 \mathrm{a}$ & $427 \mathrm{a}$ & $180 \mathrm{a}$ & $303 \mathrm{a}$ \\
\hline Spunta & $208 \mathrm{a}$ & $219 \mathrm{a}$ & $286 \mathrm{a}$ & $341 \mathrm{a}$ & $250 \mathrm{a}$ & $461 \mathrm{a}$ & $219 \mathrm{a}$ & $327 \mathrm{a}$ \\
\hline \multicolumn{9}{|c|}{ Peso fresco tubérculo (gr) } \\
\hline Newen & $77 \mathrm{a}$ & $129 \mathrm{a}$ & $413 \mathrm{~b}$ & $830 \mathrm{a}$ & $1504 \mathrm{a}$ & $2500 \mathrm{a}$ & 1999 a & $4276 \mathrm{a}$ \\
\hline Spunta & $91 \mathrm{a}$ & $139 \mathrm{a}$ & $602 \mathrm{a}$ & 909 a & $1582 \mathrm{a}$ & 3331 a & $2484 \mathrm{a}$ & $4751 \mathrm{a}$ \\
\hline
\end{tabular}

Al final del ciclo del cultivo Newen presentó en condiciones de sequía un $15 \%$ más de rendimiento total y un $27 \%$ más de rendimiento comercial que Spunta y bajo riego un $10 \%$ y un $15 \%$ más respectivamente (Tabla 3, Figura 2). La disminución del rendimiento total y comercial de Newen INTA bajo estrés hídrico en comparación a condiciones hídricas óptimas fue del 48 y $49 \%$ respectivamente. En el caso de Spunta la disminución fue de 51 y $57 \%$. Estas diferencias entre las dos variedades en el rendimiento podrían atribuirse a que el estrés hídrico afectó en mayor medida a Spunta en la etapa final de llenado de tubérculo.

Tabla 3. Rendimiento, porcentaje de materia seca y color de chips de dos variedades de papa bajo condiciones de secano y riego. Balcarce, 2014/15.

\begin{tabular}{|c|c|c|c|c|c|c|c|c|}
\hline & Secano & & \multirow{3}{*}{$\begin{array}{l}\% \\
\text { MS }\end{array}$} & \multirow{3}{*}{ chips } & \multicolumn{4}{|l|}{ Riego } \\
\hline & \multicolumn{2}{|c|}{ Rendimiento } & & & \multicolumn{2}{|c|}{ Rendimiento } & \multirow[t]{2}{*}{$\% \mathrm{MS}$} & \multirow[t]{2}{*}{ Chips } \\
\hline & Total & Comercial & & & Total & Comercial & & \\
\hline Newen & $27,12 \mathrm{a}$ & $21,85 \mathrm{a}$ & 20,20 & 6 & $52,06 \mathrm{a}$ & $42,61 \mathrm{a}$ & 20,10 & 7 \\
\hline Spunta & $22,95 \mathrm{~b}$ & $15,89 \mathrm{~b}$ & 17,18 & 5 & $47,11 \mathrm{a}$ & $36,62 \mathrm{~b}$ & 16,78 & 6 \\
\hline
\end{tabular}




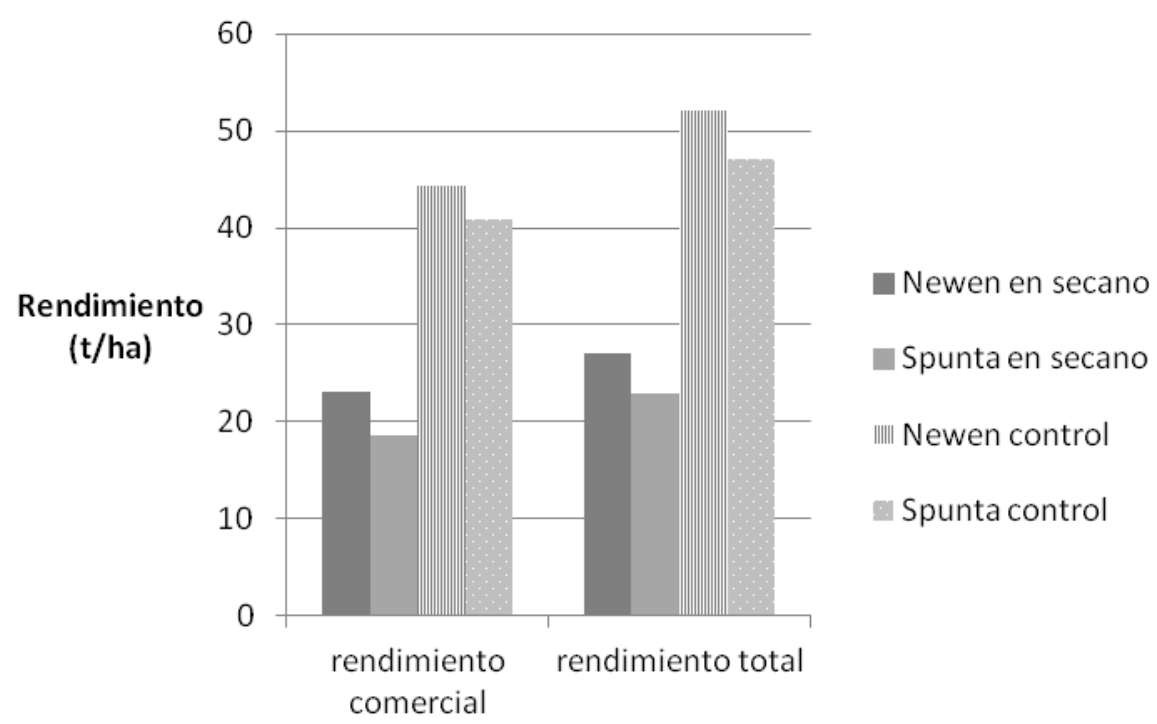

Figura 2. Rendimiento comercial y total en las variedades Spunta y Newen INTA bajo condiciones de secano y riego. Balcarce, 2014/15.

En sequía no hubo diferencias en el peso de los tubérculos menores de $50 \mathrm{~mm}$ y mayores a $90 \mathrm{~mm}$ de longitud en ambas variedades. Newen INTA presentó mayor peso de tubérculos entre 50 y $90 \mathrm{~mm}$ de longitud que Spunta (Tabla 4) Martinez y
Moreno (1992), determinaron que la formación de tubérculos pequeños por efecto de un estrés abiótico constituye un mecanismo de respuesta natural a la sobrevivencia de este cultivo.

Tabla 4. Rendimiento y porcentaje sobre el total de tamaño de tubérculos bajo condiciones de secano y riego en las variedades Spunta y Newen INTA. Balcarce, 2014/15.

\begin{tabular}{lllllllll}
\hline \multirow{2}{*}{ SECANO } & $\begin{array}{l}<0 \mathrm{~mm} \\
\text { Peso } \\
\text { t/ha }\end{array}$ & $\%$ & $\begin{array}{l}\text { Peso } \\
\text { t/ha }\end{array}$ & $\%$ & $\begin{array}{l}\text { Peso } \\
\text { t/ha }\end{array}$ & $\%$ & $\begin{array}{l}\text { Peso } \\
\text { t/ha }\end{array}$ & $\%$ \\
\hline Newen & $4,10 \mathrm{a}$ & 15,1 & $4,58 \mathrm{a}$ & 16,9 & $7,66 \mathrm{a}$ & 28,3 & $10,79 \mathrm{a}$ & 39,8 \\
Spunta & $4,31 \mathrm{a}$ & 18,8 & $1,79 \mathrm{~b}$ & 7,8 & $5,79 \mathrm{~b}$ & 25,2 & $11,05 \mathrm{a}$ & 48,2 \\
\hline
\end{tabular}

\section{RIEGO}

\begin{tabular}{lllllllll}
\hline Newen & $7,81 \mathrm{a}$ & 15 & $5,41 \mathrm{a}$ & 10,4 & $10,75 \mathrm{a}$ & 20,6 & $28,10 \mathrm{a}$ & 54 \\
Spunta & $6,20 \mathrm{a}$ & 13,2 & $0,70 \mathrm{~b}$ & 1,5 & $4,60 \mathrm{~b}$ & 9,8 & $35,60 \mathrm{~b}$ & 75,6 \\
\hline
\end{tabular}

Hubo diferencias entre las variedades con respecto al porcentaje de los tamaños de los tubérculos bajo las condiciones de riego y secano. Bajo condiciones de sequía, Newen presentó mayor porcentaje de tubérculos mayores a $50 \mathrm{~mm} \mathrm{y}$ menores a $90 \mathrm{~mm}$ de longitud que Spunta (Tabla 4). Si se compara las dos 
condiciones hídricas, Spunta disminuyó el porcentaje de los tubérculos mayores a 90 $\mathrm{mm}$ de longitud bajo condiciones de sequía en mayor medida que Newen. (48,2 vs $75,6 \%$ en Spunta y 39,8 vs $54 \%$ en Newen) Estos datos son coincidentes con Martínez y Moreno (1992), quienes determinaron que la formación de tubérculos pequeños por efecto de un estrés abiótico constituye un mecanismo de respuesta natural a la sobrevivencia de este cultivo.

En relación a la calidad culinaria, el porcentaje de materia seca y el color de las papas fritas en rodajas no se afectaron por el estrés hídrico en ambas variedades (Tabla 3).

Los resultados de esta investigación demuestran que las dos variedades se comportan diferentes ante una situación de estrés hídrico. Si bien se estimó su tolerancia ante dicho estrés de manera indirecta aún resta estudiar las bases fisiológicas y bioquímicas implicadas en dicha respuesta.

\section{Conflicto de intereses}

Los autores declaran que no existe ningún conflicto de interés en este artículo.

\section{Referencias citadas}

Argenpapa. (2016). Argentina: La producción de papa 2015/16 alcanzó las 2,43 mill. de toneladas[En línea] < http://www.argenpapa.com.ar/noticia/356 5-argentina-la-produccion-de-papa-201516-alcanzo-las-2-43-mill-de-toneladas> [consulta: Marzo 2017].

Carrillo, P.C. Moreno, J.D.; Franco, L. B.; Fierro. G. H. (2003). Manual de papa para productores.Colombia, CORPOICA. $80 \mathrm{p}$.

Coleman, W. (2008). Evaluation of wild Solanum species for drought resistance.1 Solanum gandarillasii Cardenas.
Enviromental and Experimental Botany 62:221-230.

Della Maggiora, A. (2011). Manejo del agua en el cultivo de papa. In. Curso Internacional de Producción de Papa. Facultad de Ciencias Agrarias. UNMdP.

Ekanayake, I.J. (1994). El estrés por sequía y las necesidades de riego en la papa. Centro Internacional de la Papa, Lima, Perú (Guía de Investigación CIP 30). $38 \mathrm{p}$.

FAOSTAT. (2016). [En línea] < http://faostat3.fao.org/download/Q/QC/S $>$ [consulta: Marzo 2017].

Huarte, M. A.; Capezio, S. (2013). Cultivo de papa. En: Agricultura y ganadería pampeanas. M. Cahuepe y F. Gutheim (coord.), 1a Ed. Mar del Plata, EUDEM pag 172-202. ISBN 978-9871921-11-9.

Magaña, G.I. (2013). Caracterización de genotipos de papa frente a sequía. Tesis de grado. Balcarce, Univ. Nacional de Mar del Plata. Balcarce, Argentina. 51 p.

Martinez C.A; Moreno U. (1992). Expresiones fisiológicas de resistencia a la sequía en dos variedades de papa sometidas a estrés hídrico en condiciones de campo. R. Bras. Fisiol. Veg. 4(1): 3338, 1992

Nuñez Barrios A, Ritchie J.; Smucker A. (1989). El efecto de sequía en el crecimiento, la fotosíntesis y la intercepción de luz en frijol común. [En línea] http://www.mag.go.cr/rev_meso/v09n02_ 001.pdf> [consulta: Diciembre 2017]

Ochoa,C. (1999). Las papas de Sudamérica. Centro Internacional de la Papa. Lima- Perú. pp. 501 - 503.

Olalla Mañas, F.M.,López Fuster, P; Calera Belmonte, A. (2005). Agua y Agronomía, Mundiprensa 609 p. 
Ortega Ortiz M. (2006). Respuestas fisiológicas y bioquímicas de dos especies de pinos en condiciones limitantes de humedad. [En línea] < http://www.uaeh.edu.mx/nuestro_alumna do/icap/licenciatura/documentos/Respuest as\%20fisiologicas\%20y\%20bioquimicas. pdf > [consulta: Diciembre 2017].

Pérez P.; Rodriguez Garcia R.; Zavala Garcia F.; Jasso Cantu D. (2008). Conductancia estomática y asimilación neta de CO2 en sábila (Aloe vera Tourn) bajo sequía Stomatal. [En línea] < http://www.scielo.org.mx/scielo.php?scri $\mathrm{pt}=$ sci_arttext\&pid $=\mathrm{S} 0187$ $\underline{73802010000400008}>$ [consulta: Noviembre 2017].

R Core Team. (2013). R: A language and environment for statistical computing. R Foundation for Statistical Computing, Vienna, Austria. 